\title{
Magneto-optical Trap: Fundamentals and Realization
}

\author{
K. Kowalski ${ }^{1}$, V. Cao Long ${ }^{2}$, K. Dinh Xuan ${ }^{3}$, \\ M. Glódź ${ }^{1}$, B. Nguyen Huy ${ }^{3}$, J. Szonert ${ }^{1}$ \\ ${ }^{1}$ Institute of Physics, Polish Academy of Sciences \\ Al. Lotników 32/46, 02-668 Warszawa, Poland \\ e-mail: \{krkowal/glodz/szonj\}@ifpan.edu.pl \\ ${ }^{2}$ Institute of Physics, University of Zielona Góra \\ ul. Szafrana 4a, 65-516 Zielona Góra, Poland \\ e-mail:vanlongcao@yahoo.com \\ ${ }^{3}$ Faculty of Physics, Vinh University \\ 182 Duong Le Duan, Vinh, Nghe An, Viet Nam \\ e-mail:khoadhv@yahoo.com,nghuybang@yahoo.com
}

(Received: 29 June 2010; accepted: 13 September 2010)

\begin{abstract}
Principles of laser cooling and trapping of atoms with regard to the magneto-optical trap (MOT) are described. Some details of the MOT and the accompanying measuring system, both constructed at the Institute of Physics, PAS in Warsaw, are given. Two examples of experimental results are shown.
\end{abstract}

Key words: laser cooling and trapping, Rb MOT, cold atoms, transmission spectra

\section{INTRODUCTION}

Optical cooling and trapping of neutral atoms belongs to the most effective and elegant experimental methods in atomic physics and is nowadays a widely used technique. Slow velocity, low kinetic energy, greatly reduced collisional perturbation, long interaction time, ability to store a large number of atoms at sufficiently high density - all those features cause interest in the cold atom environment as the ground for a wide variety of novel and precise experiments in the field of, for example, quantum optics, coherent phenomena, frequency standards, cold collisions or atom optics [1]. First papers on deceleration and trapping of atoms by means of laser light or confining the atoms with the magnetic field date back to several decades ago (see e.g., for earlier works [2]); we will recall an important contribution by T. Hänsch and A. Schawlow in 1975 [3], who suggested that the laser light can be used to slow down atoms. The 1980s brought such significant achievements as the first stopping of an atomic beam with a laser light by W. Phillips and H. Metcalf [4], creation of the optical molasses (OM) by Chu et al. [5] at Bell
Laboratories and construction of the magneto-optical trap (MOT) by Raab et al. [6] at the same laboratory.

In a 3D OM arrangement three pairs of mutually orthogonal and counterpropagating laser beams intersect in a vacuum chamber containing vapor of the studied element. The laser beams, tuned below the resonance, create a viscous region where the inhibiting force is exerted on the atoms. However, the radiation pressure itself does not allow for their spatial confinement. The atoms are still able to move slowly in any direction and eventually they diffuse out of the region. In order to both cool and confine atoms, the force has to be additionally position-dependent. Operation of the MOT is based on manipulations of the external and internal degrees of freedom of atoms by means of carefully prepared optical and magnetic fields [7]; the $\mathrm{OM}$ is modified by selecting the proper circular polarizations for the beams and applying a weak quadrupole magnetic field (those topics will be discussed later on).

In the first demonstration of MOT the source of the atoms was the sodium atomic beam which had been initially slowed down by the frequency swept laser 
radiation [6]. The Cs atoms were trapped in MOT for the first time in 1990, but this time the capture occurred directly from the vapor filled spectral cell at room temperature [8]. The vapor supplied traps soon turned out to be very reliable arrangements. The first Polish MOT has been operated by the group of W. Gawlik at Jagiellonian University in Cracow [9]. Currently traps in various variants (the "dark-spot" [10], two-dimensional [11, 12], allowing simultaneous trapping of two different elements $[13,14]$ or isotopes [15] and others) became the standard research tools [16]. They are used e.g. for preliminary cooling in the Bose-Einstein condensation [17-19]. In recognition of their pioneering achievements in the cooling and trapping of atoms in 1997, C. Cohen-Tannoudji, S. Chu and W. Phillips were awarded the Nobel Prize [20]. Numerous efforts were undertaken to fully understand and theoretically model the MOT [21]. At present, more than 20 different elements have been laser-cooled. The rubidium MOT described in the present work has been designed and built at the Institute of Physics, Polish Academy of Sciences in Warsaw to be one of the first devices in the country [22]. It is the main part of the apparatus for studies of the coherent and nonlinear effects in cold $\mathrm{Rb}$ atoms. In this paper we briefly review the essential cooling mechanisms related to the MOT, including both Doppler and sub-Doppler ones, and present our trap with some examples of the results. There are several excellent sources discussing many aspects of laser cooling at length [2, 23-27], our introductory treatment given below is based and benefits from these works.

Our paper is organized as follows: In Section II, an overview of the principles of laser cooling and trapping is given, including ideas of laser cooling as a momentum transfer, Doppler cooling and optical forces in optical molasses and the MOT fundamentals (Sections II.1-II.4). A brief discussion of the sub-Doppler cooling with the two polarization gradient mechanisms is given in Section II.5. In Section III, various aspects of the practical realization of the rubidium MOT are elucidated. An outline of the apparatus together with some details of the laser system, including the frequency stabilization and locking systems as well as detection and control of the experiment is given. In Section IV we give examples of results obtained with our apparatus. In Section IV.1 a high resolution spectrum of the $\mathrm{D} 2$ line of ${ }^{85} \mathrm{Rb}$ in the saturated regime is shown, and in Section IV.2 the Autler-Townes spectrum taken in the cascade 5S-5P-5D system is presented and briefly discussed. Finally, the paper is concluded in Section V with a short summary.

\section{LASER COOLING AND TRAPPING}

\section{II.1. Transfer of momentum between photon and atom}

Let us consider an idealized model of a two-level atom irradiated with a beam of resonant photons. According to the momentum conservation when a photon is absorbed its momentum $\vec{p}=\hbar \vec{k}$ ( $\vec{k}$ is the wave vector; $k=2 \pi / \lambda$ the wave number of the laser field; $\lambda$ the wavelength) is transferred to the atom retaining both its magnitude and direction (see Fig. 1(a, b)). This tiny momentum kick due to absorption of a single photon alters the velocity of an atom by recoil velocity $v_{\text {rec }}=\hbar k / M \approx 1 \mathrm{~cm} / \mathrm{s}$ (e.g. for a real $\mathrm{Rb}$ atom it is $0.6 \mathrm{~cm} / \mathrm{s}$ ) which can be compared with typical velocities of a few $100 \mathrm{~m} / \mathrm{s}$ in room temperature atoms. The absorption is followed by spontaneous emission with a natural lifetime $\tau=1 / \gamma$ of the excited state, where $\gamma$ is its decay rate (or width), and thus the atom recoils once more. Since the spontaneous emission is isotropic, the recoil of the atom associated with this process is in a random direction, thus there is no net change of momentum on average. Thus overall the change of the atomic momentum is solely the effect of absorption (see Fig. 1(c)). The force $\vec{F}=d \vec{p} / d t$ acting on the atom is directed along the laser
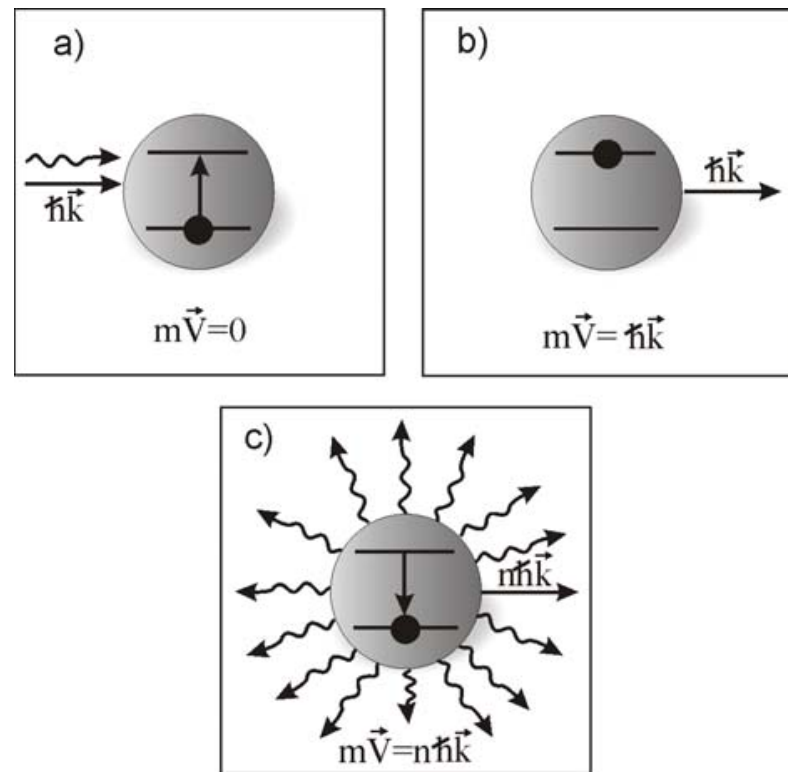

Fig. 1. A simplified depiction of the light pressure acting on the two-level atom. (a) the resonant photon approaches an atom of mass $M$ in its ground state and in rest; (b) as a result of absorption the atom, now in the excited state, gains a momentum kick, $M \vec{v}=\hbar \vec{k} ;$ (c) recoil momentum due to the isotropic spontaneous emission averages out over many absorption/emission cycles thus, after $n$ cycles the atom gains momentum $M \vec{v}=n \hbar \vec{k}$ along the propagation direction of incoming photons. The wavy arrow is for photon, the thick one for momentum 
beam. Its value increases with intensity of the incoming light until the stimulated emission begins to play a role. Higher-intensity produces faster absorption, but causes also equally fast stimulated emission. The photon emitted by the stimulated emission moves in the same direction as the stimulating photon. It follows that the momentum transfer in the process of the stimulated emission is directed opposite to the momentum transfer during the absorption. Thus the total change of atomic momentum in such a sequential process equals zero. Therefore, the resultant (de/ac)celeration cannot exceed value $\vec{a}_{\max }=\hbar \vec{k} f \gamma / M$, (where $M$ is the mass of the atom and $f$ is the fraction of time it spends in an excited state) [28]. In a high intensity beam the atom spends half of its time in each of the states, thus $f_{\max }=1 / 2$. With narrow linewidth lasers easily available the scattering rate of $10^{7} \mathrm{~s}^{-1}$ or more absorption acts/s is realistic. In such condition an atom moving in the direction opposite to the photon beam could be decelerated very efficiently. Simple evaluation shows e.g. that for the $\mathrm{Rb}$ atom $a_{\text {max }} \sim 10^{4} \mathrm{~g}$ where $g$ is an earth gravity constant. Taking typical initial thermal velocity, the time to halt an atom amounts to $\Delta t \sim 1 \mathrm{~ms}$. Thus with lasers one can brake really hard.

\section{II.2. Doppler cooling and optical molasses}

If a two-level atom of velocity $\vec{v}$ propagates agains $(\vec{k} \vec{v}<0)$ a low intensity laser beam of frequency $\omega_{L}$ tuned slightly below the atomic resonance of frequency $\omega_{0}$ $\left(\omega_{L}<\omega_{0}\right)$, then the travelling atom sees the Doppler shifted frequency

$$
\omega=\omega_{L}-\vec{k} \vec{v}
$$

which is higher and thus closer to atomic resonance. For red detuning the atom will see this frequency to be at a resonance if the relation: $\omega_{L}(1+v / c)=\omega_{0}$ holds. Then, photons will be absorbed and the atom slows down. On the contrary, if the atom co-propagates with the laser beam $(\vec{k} \vec{v}>0)$ the Doppler shifted frequency increases its distance from resonance and consequently the absorption probability is even further reduced. Thus, the atom undergoes an asymmetric influence by the laser beam depending on the direction of its motion $(\vec{v})$. It can be noted that atoms of velocity $\vec{v}$ (with spread determined by $\gamma$ ) satisfy the condition

$$
\omega_{L}-\vec{k} \vec{v}=\omega_{0}
$$

and are slowed down. By using three intersecting orthogonal pairs of counter-propagating laser beams, the above elucidated slowing effect can be extended to 3D [3]. Each atom moving in this field will be acted by a deceleration force much stronger than the acceleration one. Both forces equalize only if the atom is not moving. Such environment is called optical molasses (OM) [5]. Atoms in OM are free to move around (via diffusion) anywhere within the region of overlapping beams, before they slowly leave the region. At first glance it might be guessed that the residual kinetic energy of an atom in OM equals the photon recoil; however, it is not the case. Its temperature (energy) in general depends on detuning [26] and in minimum reaches limit $T_{D}=\hbar \gamma / 2 k_{\mathrm{B}}$ which is much higher than the recoil temperature $T_{\text {recoil }}=\hbar^{2} k^{2} /\left(2 M k_{\mathrm{B}}\right)$. The former is called the Doppler limit (see Section II.4 for further discussion).

In the moving atom's reference frame, the net force $\vec{F}$ exerted by a single laser beam depends directly on the scattering (excitation) rate of photons $\Gamma_{S C}($ e.g., [24]):

$$
\vec{F}=\hbar \vec{k} \Gamma_{S C},
$$

with

$$
\Gamma_{S C}=\frac{\gamma S / 2}{1+S+\left[2\left(\delta+\omega_{D}\right) / \gamma\right]^{2}}
$$

where $S=I / I_{S}$ the saturation parameter, $I$ the light intensity, $I_{S}=\pi h c \gamma / 3 \lambda^{3}$ the saturation intensity of the transition; $\omega_{D}=-\vec{k} \vec{v}$ the Doppler shift; $\delta=\left(\omega_{L}-\omega_{0}\right)$ the detuning. $\Gamma_{S C}$ has a Lorentzian dependence on velocity.

Consider now two counterpropagating beams weak enough so that their effects taken separately are additive, and those caused simultaneously by both beams can be neglected. Then the total light pressure force $F_{O M}$ from the two beams is

$$
\vec{F}_{O M}=\vec{F}_{+}+\vec{F}_{-},
$$

where

$$
\vec{F}_{+,-}= \pm \frac{\hbar \vec{k} \gamma}{2} \frac{S}{1+S+\left[2\left(\delta \mp \omega_{D}\right) / \gamma\right]^{2}}
$$

In Fig. 2 the component forces $\vec{F}_{+}$and $\vec{F}_{-}$as well as their sum $\vec{F}_{O M}$, calculated for representative cooling parameters $(\delta=-1.3 \gamma, \quad S=3)$ are shown as a function of velocity. As can be seen for all velocities, the force exerted on an atom is damping its movement. The atomic motion is damped if the laser is tuned below an atomic resonance. There is also a relatively narrow range of velocities where the force on velocity dependence is linear. Assuming the atoms are slow $\left(\left|\omega_{D}\right|=|\vec{k} \vec{v}|<<\gamma\right)$ terms of second and higher orders in $\omega_{D}$ of the respective expansion can be neglected and one obtains an approximate expression [25]: 


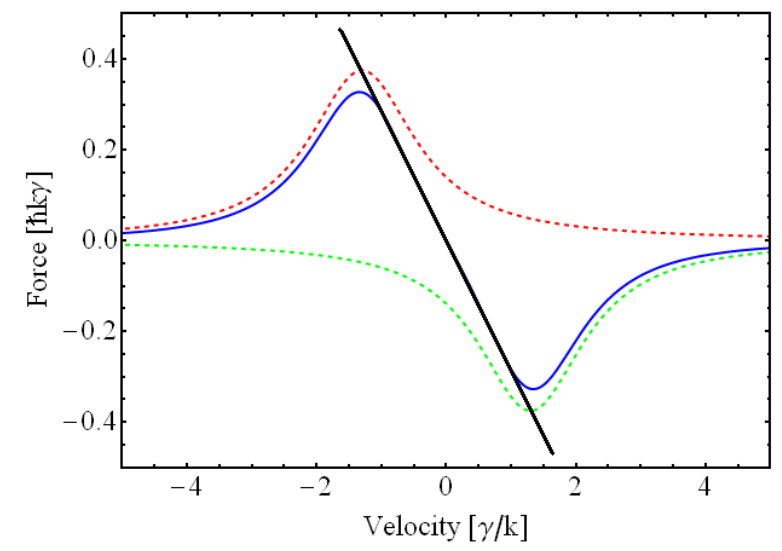

Fig. 2. Dependence of the optical damping forces eqns. (5), (6) on velocity for 1D OM calculated for the detuning $\delta=-1.3 \gamma$ and the saturation $S=3$. The dotted traces show the components $\vec{F}_{+}$(red trace) and $\vec{F}_{-}$(blue trace) from each beam while the solid curve (blue trace) is their sum. The straight line (black trace) shows the velocity range where proportionality holds
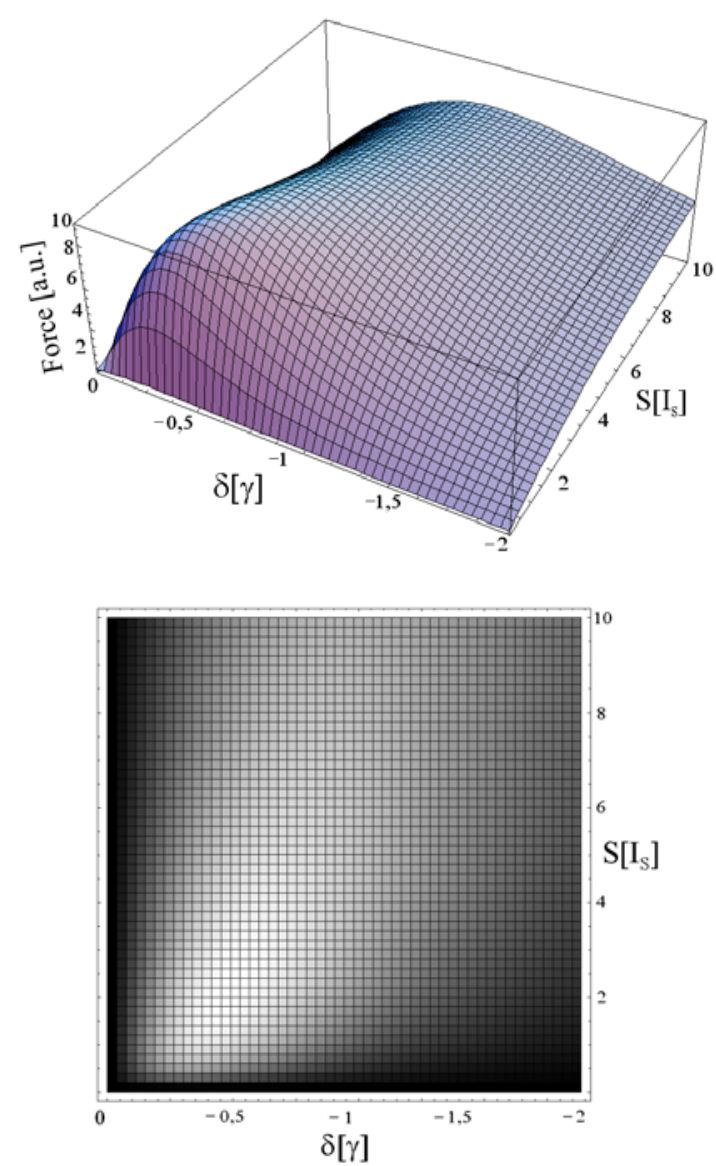

Fig. 3. The damping force $F_{O M}$, Eq. (7), exerted on an atom as a function of the detuning $\delta$ and the saturation $S$; (a) 3D plot; b) 2D map, with brighter points corresponding to the larger force. $\delta$ in units of the decay rate $\gamma, S$ in units of the saturation intensity $I_{S}$

$$
\vec{F}_{O M} \cong \frac{8 \hbar k^{2} \delta S \vec{v}}{\gamma\left[1+S+(2 \delta / \gamma)^{2}\right]^{2}},
$$

or in short form:

$$
\vec{F}_{O M} \cong-\beta \vec{v} .
$$

where in the (friction) parameter $\beta$ all constants and parameter values of Eq. (7) are included. Thus, indeed as it can be seen in Fig. 2, for slow atoms $F_{O M}$ is proportional to velocity. It resembles the viscous damping. It is why this technique is called $O M$. The dependence of $F_{O M}$ Eq. (7) on the cooling parameters $\delta$ and $S$ is depicted in Fig. 3; in (a) as a $3 \mathrm{D}$ plot and in (b) the same dependence is shown as a 2D map where the brighter points symbolize the larger force value. As can be seen from (7) e.g. with $S=1$ the maximum force is reached when $\delta=-\gamma / 2$. Depending on a sign of detuning the force can be attractive or repulsive. Cooling is possible only for negative values of $\delta$, in the opposite case heating occurs and the atom gains energy. It should be noted that the resonance radiation pressure force of the type Eq. (8) alone is not able to provide stable trapping of atoms [29].

\section{II.3. 1D model of the MOT}

Let us consider a 1D model of the MOT. It consists of two laser beams of opposite circular polarization (right $\sigma^{+}$and left $\sigma^{-}$) counterpropagating along the $z$-axis, the coaxial inhomogeneous magnetic field $\vec{B}$ of quadrupole symmetry (Fig. 4) and the atoms of simplest level structure with hyperfine components $F=0$ and $F^{\prime}=1$ for the ground and excited states, respectively. The $\sigma^{+}$(or $\sigma^{-}$) circularly polarized beam causes only transitions with $\Delta m_{F}=+1$ (or $\Delta m_{F}=-1$ ) (Fig. 5). $\vec{B}$ changes linearly with $z$ and its sign alters in the trap center (Fig. 6). Under the influence of the magnetic field the degeneracy is lifted (Zeeman effect) and

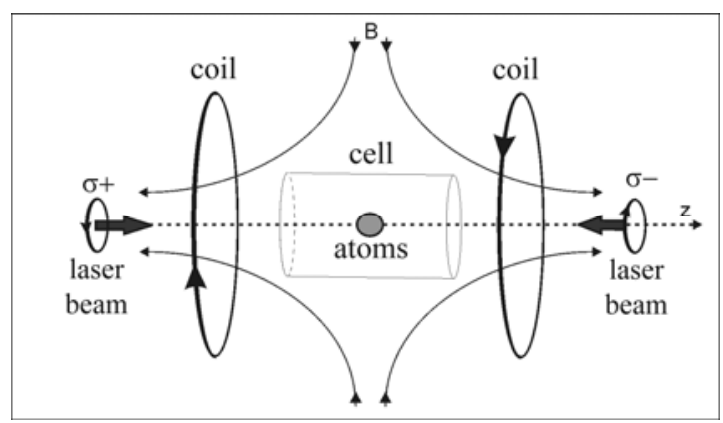

Fig. 4. Arrangement for a MOT in 1D. Atoms in the field of two counterpropagating beams and the magnetic field of quadrupole symmetry with zero in the trap centre 
each hyperfine state $F$ splits in $2 F+1$ sublevels. The Zeeman shift of the sublevel is proportional to its magnetic quantum number $m_{F}$ and to the $B$ field:

$$
\Delta E_{m_{F}}=g_{F} \mu_{B} B m_{F},\left(m_{F}=0, \pm 1, \ldots, \pm F\right),
$$

where: $g_{F}-$ Lande factor, $\mu_{B}-$ Bohr magneton, $B-$ intensity of the magnetic field.

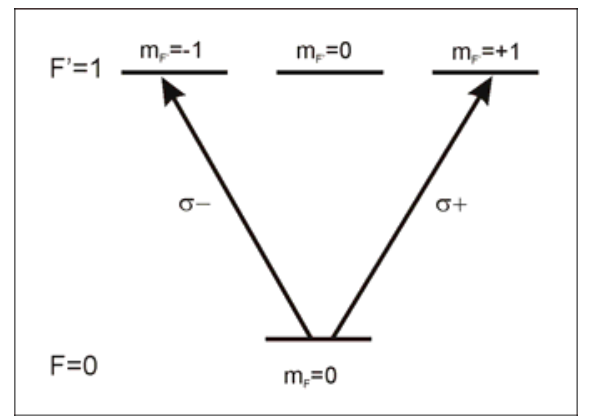

Fig. 5. Selective absorption of the circularly polarized light

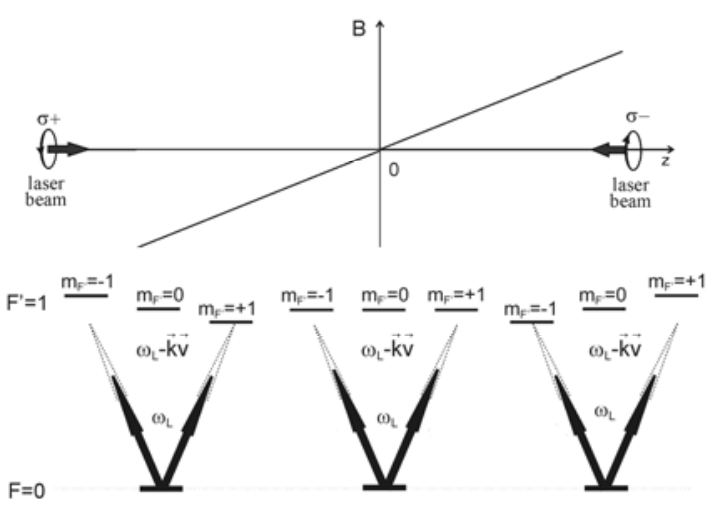

Fig. 6. Atom of a simple level structure $\left(F=0, F^{\prime}=1\right)$ in the 1D MOT. Zeeman shifts of the sublevels in the $B$-field of constant gradient $\partial B / \partial z$ are shown. The black arrows denote the laser frequency red-detuned from the resonance; the dotted arrows are for the Doppler modified frequency

In the inhomogeneous magnetic field of constant gradient $\partial B / \partial z$ the sublevels' splitting changes linearly with $z$ (Fig. 6). We consider an atom situated to the left of the reference frame. The $B$ field in this region has a negative value. The beam incident from the left and propagating toward the trap center is $\sigma^{+}$polarized. When the cooling condition is satisfied (each beam tuned below zero field atomic resonance), the transitions between the sublevels $F=0$ and $F^{\prime}=1$ are caused mainly by the $\sigma^{+}$light. Momentum transfer along the incident $\sigma^{+}$beam toward the trap center occurs. The counterpropagating $\sigma^{-}$beam is significantly more detuned from the resonance in this region. The momentum transfer induced by this beam repelling the atom out of the trap center is very small. As a result, the atom is pushed toward the field free $(B=0)$ region. To the right of the trap, the mirror situation happens. However, now the $\sigma^{-}$beam is tuned significantly closer to the resonance than the $\sigma^{+}$beam, so the momentum transfer occurs in the direction of the $\sigma^{-}$beam. Thus, two forces act on the atom, their sum is directed always toward the center of the trap and equals zero in this point.

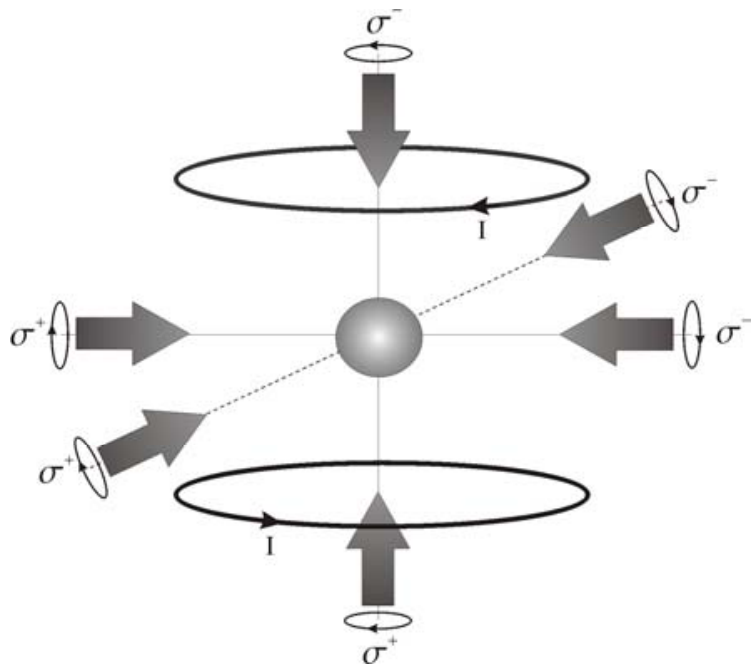

Fig. 7. Scheme of a 3D MOT. Thick arrows denote three pairs of the circularly polarized cooling laser beams counter-propagating along three coordinate axes. Big loops of current $I$ flowing in opposite directions generate magnetic field of spherical quadrupole symmetry

Allowing for the $B$ field in an OM Eq. (6) modifies. It assumes the form:

$$
\vec{F}_{+,-}= \pm \frac{\hbar \vec{k} \gamma}{2} \frac{S}{1+S+\left[2\left(\delta \mp \omega_{D} \mp \mu^{\prime} B / \hbar\right) / \gamma\right]^{2}},
$$

where $\mu^{\prime}=\left(g_{e} m_{e}-g_{g} m_{g}\right) \mu_{B}$ the effective magnetic moment for the cooling transition; $g_{e}\left(g_{g}\right)$ Lande factor of the excited (ground) state; $m_{e}\left(m_{g}\right)$ magnetic number of the excited (ground) state; $\omega_{D}=-\vec{k} \vec{v}$ the Doppler shift; $\left|\omega_{Z}\right|=\mu^{\prime} B / \hbar$ the Zeeman shift.

Here we allowed the magnetic structure in both states. Note that $B=B(z)$. Assuming that both $\left|\omega_{D}\right|$ and $\left|\omega_{Z}\right|$ are small as compared to $\delta$, the denominator of Eq. (10) can be expanded, as earlier in Eqns. $(5,6)$, keeping only linear terms, the approximate form reads

$$
\vec{F}_{M O T} \cong-\beta \vec{v}-\xi \vec{r},
$$

where $\beta$ is the same as in Eq. (8) and the damping constant $\xi$ arises from the similar functional dependence of $F_{M O T}$ on both $\omega_{D}$ and $\omega_{Z}$, and is given by 


$$
\xi=\frac{\partial B}{\partial z} \frac{\mu^{\prime} \beta}{\hbar k}
$$

Note that Eq. (11) is identical to the damped oscillator one. The 3D generalization of the $1 \mathrm{D}$ model is rather natural. The atoms are irradiated by pairs of opposing laser beams propagating along three Cartesian axes. Along the $z$-axis two coaxial identical coils centered with beams' crossing point and carrying opposite currents are placed. The created magnetic field has the spherical quadrupole symmetry, with gradient along the $z$-axis twice as big as that in the $x y$ plane. Provided a proper choice of various parameters (including the selection of the right closed atomic transition), such configuration allows very efficient slowing and confining of the real atoms. In the real MOT field of $\partial B / \partial z$ gradient of the order $10-20 \mathrm{G} / \mathrm{cm}$ is used.

\section{II.4. Doppler cooling limit}

In Section II.1 the cooling mechanism has been introduced, in which atoms are cooled by resonant photons via the momentum exchange. It has been emphasized that the isotropic character of the spontaneous emission averages out and therefore does not influence the atomic momentum. It could seem that in the end the initially moving atoms will decelerate rapidly to zero velocity. However, it was argued above that the momentum kicks resulting from photon recoil impose its lower limit which is nonzero. This can be described as a random walk in momentum space with $\hbar k$ step size. The mean square atomic momentum $<p^{2}>\neq 0$, it changes with each act of absorption or the spontaneous emission. 'The temperature results from an equilibrium between laser cooling and the heating process arising from the random nature of both the absorption and emission of photons' [20]. To evaluate the equilibrium temperature we consider the rates of processes which cause atoms to heat (by the random walk in momentum space) and to cool (by the cooling force exerted by photons as discussed above). The rate at which the energy is removed by cooling equals the product of the force (Eq. (8)) and the velocity

$$
\left(\frac{d E}{d t}\right)_{\text {cooling }}=\vec{F}_{O M} \vec{v}=-\beta \vec{v}^{2} .
$$

It is proportional to the velocity squared and thus the kinetic energy. In contrast, the heating rate depends on the total photon scattering rate only. For one beam this rate is given by Eq. (4). However, since in an equilibrium atoms move very slowly $\left(\omega_{D}=|k v|<<\gamma\right)$ the Doppler shift can be neglected. Thus the rate of change of $<p^{2}>$ amounts to:

$$
\frac{d}{d t}<p^{2}>=\hbar^{2} k^{2} \Gamma_{S C}^{\prime},
$$

After each cycle of absorption and spontaneous emission the atom gains the kinetic energy:

$$
E_{K}=\frac{<p^{2}>}{M} .
$$

The rate of its change is

$$
\left(\frac{d E}{d t}\right)_{\text {heating }}=\frac{1}{M} \frac{d}{d t}<p^{2}>=\frac{1}{M} \hbar^{2} k^{2} \Gamma_{S C}^{\prime} .
$$

At an equilibrium

$$
\left(\frac{d E}{d t}\right)_{\text {cooling }}=\left(\frac{d E}{d t}\right)_{\text {heating }}
$$

From Eqns. (13), (16), (17) we derive:

$$
v^{2}=\frac{1}{\beta} \frac{1}{M} \hbar^{2} k^{2} \Gamma_{S C}^{\prime}=\frac{3 \hbar \gamma^{2}\left(1+S+4 \delta^{2} / \gamma^{2}\right)}{8 M \delta}
$$

Using the equipartition theorem, the effective temperature can be inferred as

$$
T=\frac{M v^{2}}{3 k_{B}}=\frac{\hbar \gamma^{2}\left(1+S+4 \delta^{2} / \gamma^{2}\right)}{8 k_{B} \delta} .
$$

By taking the derivative with respect to $\delta$ and performing the usual procedure the lowest temperature limit can be determined. For the optimum value $\delta_{\text {opt }}=-\gamma / 2$ it amounts to:

$$
T=\frac{\hbar \gamma(2+S)}{4 k_{B}}
$$

In the low intensity limit the minimum called the Doppler limit is obtained:

$$
T_{D} \cong \frac{\hbar \cdot \gamma}{2 k_{B}}
$$

It is noteworthy that $T_{D}$ depends only on $\gamma$ - the inverse lifetime (the width) of the excited state used in the cooling. The dependence of the temperature $T$ on the detuning $\delta$ calculated for $\gamma=2 \pi \times 5.9 \mathrm{MHz}$ corresponding to the ${ }^{85} \mathrm{Rb}$ $\left(5 \mathrm{P}_{3 / 2}\right)$ is shown in Fig. 8. At $\delta=-\gamma / 2(\sim 2 \pi \times 3 \mathrm{MHz})$ $T_{D}=143 \mu \mathrm{K}$ which corresponds to velocity of $12 \mathrm{~cm} / \mathrm{s}(e . g$., for $\left.\mathrm{Na} T_{D}=240 \mu \mathrm{K}\right)$. Typically $T_{D}$ is few $100 \mu \mathrm{K}$ corresponding to velocities of ca $10 \mathrm{~cm} / \mathrm{s}$. The transitions of narrow width or of the width influenced externally are considered as candidates for use in the Doppler cooling with the aim to approach the recoil limit (Eq. (22)) of the temperature [30]. 


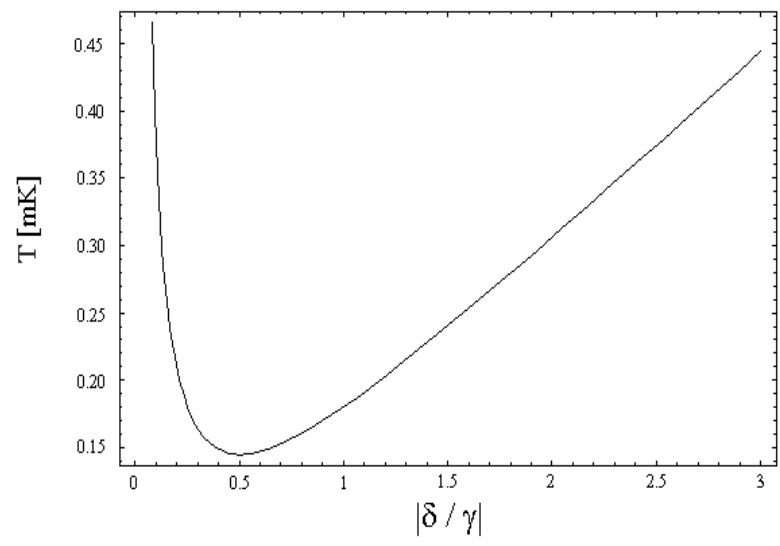

Fig. 8. Dependence of temperature on detuning of the cooling laser calculated for ${ }^{85} \mathrm{Rb}$ from the Eq. (19). The minimum $T_{D}=143 \mu \mathrm{K}$ is achieved at the detuning $\delta=-\gamma / 2$

\section{II.5. Sub-Doppler Cooling Mechanisms - Polarization Gradient Cooling}

It was widely accepted that the Doppler limit $T_{D}$ poses the lowest temperature which can be obtained in OM. Surpisingly, P.D. Lett et al. measured temperature in the laser cooled sodium OM which was six times lower than the expected limit [31]. This triggered theoretical efforts to understand the discovery. The essential new contribution introduced by these works relies in the understanding of the specific role that the multilevel structure of the atom and the polarization of the light play in the laser cooling [32, 33]. The new approach is known as a sub-Doppler cooling and includes several different mechanisms. Below are two examples of such mechanisms which allow temperatures below the Doppler limit. They are known as the polarization gradient cooling mechanisms [32, 33].

The atom in a nearly resonant field, aside from absorption, is also subject to light induced shifts of its energy levels known as the ac-Stark shift. In the limit of low light intensity it amounts to $\Delta E_{g, e} \approx \pm \hbar \Omega^{2} / 4 \delta$ with $\Omega^{2}=S \gamma^{2} / 2$ and $\Omega$ the Rabi frequency. Since it is proportional to the intensity, $\Delta E_{g, e}$ is known as the light shift. The light shift can result in a gradient force (the dipole force) on an atom when the intensity varies in space. Such situation can be found e.g., in the strong focusing and the Gaussian intensity profile of the beam. Another case of interest here is the spatial intensity dependence between nodes and antinodes of a standing wave. Consider now a simple model of an atom with the ground (excited) state angular momentum of $J_{g}=1 / 2 \quad\left(J_{e}=3 / 2\right)$ placed in a field of two counterpropagating laser beams of the same frequency and orthogonal, polarizations and propagating with velocity $v$ along the $z$ axis. We briefly consider two cases of (a) orthogonal linear polarizations (lin $\perp$ lin case) and (b) orthogonal circular polarizations $\left(\sigma^{+} \sigma^{-}\right.$case $)$.

\section{lin $\perp$ lin case}

The interfering beams form a standing wave of polarization which varies cyclical with $z$ coordinate. Over the distance of $\lambda / 2$ the polarization of the resulting field alternates from the linear $\pi$ (at $0^{\circ}$ ), over circular $\sigma^{-}$, linear (at $90^{\circ}$ ), opposite circular $\sigma^{+}$to the linear $\pi\left(\right.$ at $180^{\circ}$ ), and the cycle repeats itself with the period of $\lambda / 2$. (Note that the amplitude of the field remains unchanged here). Thus the field has a gradient of polarization. Under the influence of the light field the population distribution of the magnetic sublevels of the ground state becomes modified. It occurs depending on the actual polarization acting on the atom (due to selection rules of $\Delta m_{F}=0,+1,-1$ for $\pi, \sigma^{+}$and $\sigma^{-}$polarized light, respectively) as a result of multiple cycles of absorption and subsequent fluorescence. For $z$-coordinate where the effective polarization is $\sigma^{+}$the population is transferred entirely to the $m_{g}=+1 / 2$ sublevel, while for $\sigma^{-}$the population is transferred entirely to the $m_{g}=-1 / 2$ sublevel. This process is known as an optical pumping (In general case the distribution depends on the coupling strength of the transitions which are proportional to the respective Clebsch-Gordan (C-G) coefficients squared). The light force active in this process is the same as in the earlier discussed two-level Doppler case. Because the atom travels along $z$, it has to adapt its population distribution each time it changes the polarization acting on it. However, as stated in a number of lines above, each level undergoes a light shift of a magnitude dependent also on the coupling strength (and thus on polarization and the magnetic number via respective $C-G$ coefficient) of the transition. Both modifications (i) the population distribution and (ii) the respective light shifts change simultaneously as the atom travels along the polarization gradient. In general for negative (positive) detuning the ground level shifts into lower (higher) energies and the opposite holds for the excited level. Thus for $\delta<0$ (red detuning) the highly populated ground state sublevel 'repels' from its excited 'partner', thus increasing the resonance energy needed for the transition. The light-shifted energies of the ground state sublevels $m_{F}=1 / 2$ and $m_{F}=-1 / 2$ oscillate in space with the same periodicity as the polarization does. Moreover, the light shifts of both sublevels $m_{F}=1 / 2$ and $m_{F}=-1 / 2$ are not the same because the respective C-G coefficients differs for each circular polarizations. The spatial dependence of the light shifts of the ground-state sublevels is shown in Fig. 9. Energy of each sublevel changes with $z$ as a sine function with the $\pi$-phase shift between them. The sublevels have the same shifts where 
the polarization of the resulting field is linear, because for this polarization the squares of the C-G's are equal. For the $\sigma^{+}$polarization the squared C-G of the sublevel $m_{F}=1 / 2$ is three times larger than that of the sublevel $m_{F}=-1 / 2$. In the region where the light polarization becomes $\sigma^{-}$the opposite occurs, the shift of $m_{F}=-1 / 2$ is three times larger. In Fig. 9 the cooling mechanism is illustrated. As the atom in the $m_{F}=+1 / 2$ propagates along $z$ the potential energy changes along with the polarization, the atom is climbing a potential hill (at $z=\lambda / 8$ ) losing its kinetic energy and gaining the potential one, at the hill top it is optically excited with $\sigma^{-}$light and as a result of spontaneous emission returns back to the $m_{F}=-1 / 2$ level which is now of the lower energy. Thus the emitted photon carries out more energy than the absorbed one. As the atom propagates further the reverse process starts with the $m_{F}=-1 / 2$ level populated and situated in the potential valley. Thus in a single absorption/emission cycle the atom loses energy equal the light induced splitting between both levels. The cooling will be no longer possible when the kinetic energy is lower than the height of the hill. The kinetic energy removed from the atom is radiated out. The above mechanism is known in the literature as Sisyphus cooling, and was presented in [32]. It should be underlined that it needs a multilevel structure of the ground state and works over a narrow range of velocities only.

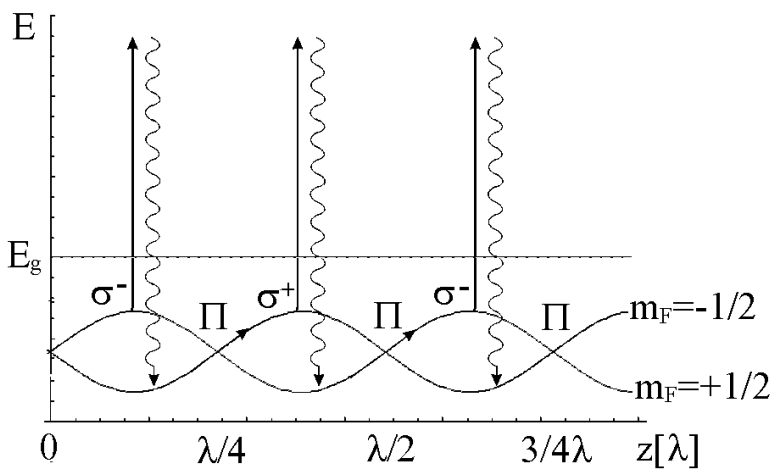

Fig. 9. Sisyphus cooling. Periodic dependence of the light-shifted ground levels' energy in the field of two counterpropagating beams of linear, orthogonal polarizations. The correlated changes of the effective polarization $\left(\sigma^{-}, \pi, \sigma^{+}\right)$are shown. The dotted line denotes the field-free energy $\left(E_{g}\right)$. Full arrow denotes absorption and wavy one fluorescence

\section{$\sigma^{+} \sigma^{-}$case}

The interfering beams of orthogonal circular polarizations form a standing wave of polarization which is linear all along the $z$ axis and its plane rotates periodically at full $2 \pi$ over the distance of $\lambda$ (the trace resembles the helix or the screw). Thus, as above, the field has a polarization gradient, but its amplitude stays constant. An interaction of the moving atom with the light field here is more involved than in the former case and the mechanism is radically different [32]. Since the laser intensity is constant and the polarization is linear, all ground levels are shifted in the same way and they do not vary with $z$. It follows that while in the lin $\perp$ lin case the dipole force is present, here it does not. Therefore, the Sisyphus effect is not possible. However, instead of the way it was established in [32], the atomic motion-induced orientation among the levels of the ground state is created. (The simplest model for which it can be demonstrated is the $J_{g}=1\left(J_{e}=2\right)$ system.) As follows the counterpropagating $\sigma^{+}$and $\sigma^{-}$beams are absorbed with different efficiencies, which results in an unbalanced radiation pressure (similarly as in the Doppler cooling) when the atom moves. The damping force is established. It constitutes an important difference between the Sisyphus mechanism and the $\sigma^{+} \sigma^{-}$case. Although it seems to be similar to the Doppler cooling, the $\sigma^{+} \sigma^{-}$polarization gradient cooling is different. It uses the unequal distribution (orientation) of the Zeeman levels while the Doppler cooling utilizes the Doppler shift of the beam frequency.

Cooling with the polarization gradient (Sisyphus cooling) is possible only for atoms initially pre-cooled by the Doppler cooling, atoms have to be sufficiently slow. The time needed for optical pumping to take effect $\tau=1 / \gamma$ should be shorter than the time necessary for the atom to travel the distance of $\lambda / 4$, which determines the characteristic velocity of $v=\gamma / k$. The faster atoms will not be affected with the Sisyphus cooling. The other of the discussed mechanisms has no such restriction. Both of the above discussed mechanisms can produce the ultra-low temperatures (of the order of few $100 \mathrm{nK}$ ) with a characteristic limit related to the recoil momentum. It is known as the recoil temperature

$$
T_{\text {recoil }}=\frac{\hbar^{2} k^{2}}{2 M k_{B}},
$$

e.g., for ${ }^{85} \mathrm{Rb}$ which is of interest here, this temperature amounts to $T_{\text {recoil }}=185 \mathrm{nK}$. It can be noted that in the real 3D OM the complex effective field polarization pattern causes that both discussed mechanisms are important.

By selecting the element and/or isotope the importance of different cooling mechanisms can be studied. In contrary to alkali metals, the Group II elements (like e.g., Ca, Sr) have two valence electrons and thus zero electronic angular momentum $\left(J_{\mathrm{g}}=0\right)$ in the ground state. Using even isotopes (of $I=0$ nuclear spin) one can study the Doppler cooling without the interfering effect of the Sisyphus cooling mechanism. In various odd isotopes, on the other hand, $I \neq 0$ and the Sisyphus cooling can be studied as a function of a different number of ground state sublevels. 
Ytterbium of III Group apart from $J_{g}=0$ has an advantage of several isotopes of $I=0, I=1 / 2$ and $I=5 / 2$, thus allowing comparison to be performed for a single element e.g., a comprehensive study was reported in [34]. Other cooling mechanisms leading to the sub-Doppler cooling are known just to mention two of them: cooling by Raman resonances [35] or the velocity selective coherent population trapping [36]. Both allow temperatures below the recoil limit.

\section{REALIZATION OF THE MOT}

\section{III.1. Introduction}

For their simple structure alkali metal atoms are very popular objects of the study. The ground to the first excited state transition frequency is the visible or near infrared range. The light appropriate for all alkalis except the sodium can be produced using the cost effective diode lasers. Their handling, operation, frequency stabilization methods are well elaborated. The trap construction is well known and not very sophisticated. Therefore, alkali atom based MOTs are widely used and offer the way to produce atomic samples with temperatures below $1 \mathrm{mK}$, at a relatively modest cost. The essential part of the diode laser system for spectroscopy of cold $\mathrm{Rb}$ atoms designed and built at the IP PAS is a standard six-beam rubidium MOT in a glass chamber, similar to the one first reported in Ref. [8]. In the presence of an adequate magnetic field, the velocity- and position-dependent forces cause both cooling and confinement of atoms, which become trapped at the $B=0$ point.

Below, the experimental setup, the practical realization of our MOT system is briefly presented. While it can be applied for both the ${ }^{85} \mathrm{Rb}$ and ${ }^{87} \mathrm{Rb}$ isotopes, to simplify the presentation we will limit to the more abundant ${ }^{85} \mathrm{Rb}$ isotope. Besides the MOT lasers, other extended cavity diode lasers (ECDLs) are used for spectral studies. The system is equipped with digital laser frequency tuning-, experiment control- and data acquisition- systems. Our apparatus was earlier described in [22], and some details are repeated here.

\section{III.2. Description of the trap}

In Fig. 10, the hyperfine structure of the D2 line of ${ }^{85} \mathrm{Rb}$ used in the process is shown, as well as the transitions, at $780 \mathrm{~nm}$, induced by the two MOT lasers. In order to achieve cooling \& trapping the first (strong) laser is slightly red-detuned against the $5 \mathrm{~S}_{1 / 2}(F=3) \rightarrow 5 \mathrm{P}_{3 / 2}\left(F^{\prime}=4\right)$ cooling transition. This transition is closed, an atom excited into the $F^{\prime}=4$ level spontaneously returns back to the starting $F=3$ ground level. However, despite significant frequency mismatch there is still a non-negligible chance for an off-resonant excitation of another transition to the $F^{\prime}=3$ level as well. In that case the decay to $F=2$ and $F=3$ follows. Even if weak after short time, the optical pumping will transfer all available atoms into the $F=2$ level. The cooling will become impossible. The repumping laser, resonant with the $5 \mathrm{~S}_{1 / 2}(F=2) \rightarrow 5 \mathrm{P}_{3 / 2}\left(F^{\prime}=3\right)$ transition serves to return atoms, which leaked to the $5 \mathrm{~S}_{1 / 2}(F=2)$ state, back to the trapping cycle. The general optical scheme of the MOT is depicted in Fig. 11.

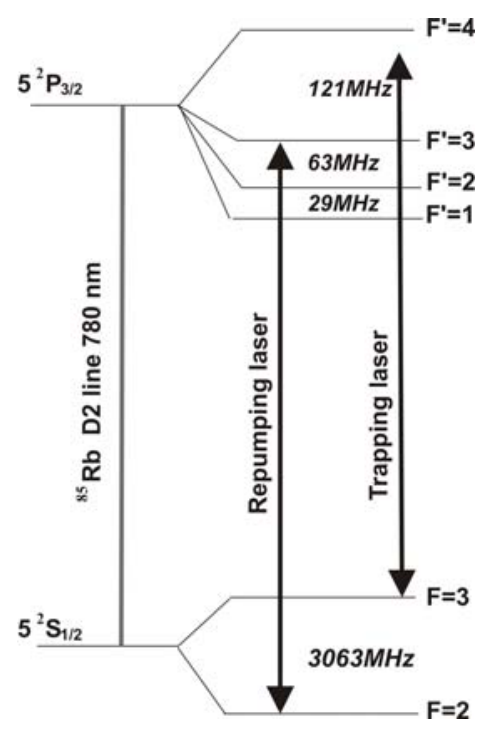

Fig. 10. The relevant levels of ${ }^{85} \mathrm{Rb}$ and transitions involved in the operation of the MOT. The hyperfine level spacing are given

The MOT chamber (typical size of $80 \mathrm{~mm}$ ) is made of quartz glass and equipped with six windows to accept the laser beams. It is connected to another glass cell containing three resistively heated $\mathrm{Rb}$ dispensers (SAES Getters) serving as a thermal source of $\mathrm{Rb}$ atoms. The chamber is permanently connected to a high-vacuum system based on a $20 \mathrm{l} / \mathrm{s}$ (magnetically shielded) ion pump and a titanium sublimation pump. The residual pressure, as determined from the current of a continuously working ion pump is less than $10^{-8}$ Torr. Magnetic coils of $80 \mathrm{~mm}$ in diameter and separated by 1.25 radii in an anti-Helmholtz configuration produce a field of quadrupolar symmetry (with gradient up to $40 \mathrm{Gs} / \mathrm{cm}$ ) necessary for confinement of atoms. Three additional orthogonal pairs of coils surround the chamber to compensate the stray magnetic fields in the trap center. Two commercial extended cavity diode lasers (ECDLs) (type DL100, TuiOptics-Toptica) (DL1, DL3), and a home-made amplifier (DL2) comprise the MOT laser system (1 MHz linewidth). Each laser is 
protected with a double $60 \mathrm{~dB}$ optical isolator (Gsänger) to avoid optical back reflections into the laser cavity. The ECDLs are built in the Littrow configuration with a diffraction grating placed in front of the laser in such a way that the 1st order diffracted beam is reflected back into the laser diode, while the 0th order beam forms the laser output $[37,38]$. The role of the grating is to control, with the help of fine-adjustment screws and a piezoelectric transducer (PZT), the frequency of the emitted light and to narrow its linewidth. The lasers DL1 and DL2 work in a master-slave injection locking configuration [39] providing the trapping beam, while DL3 is the repumping laser. Both trapping $(40 \mathrm{~mW})$ and repumping $(15 \mathrm{~mW})$ beams are combined to co-propagate, spatially reshaped to be nearly circular with anamorphic prism pair, and expanded with a telescope to the diameter of $17 \mathrm{~mm}$. Inside the telescope a shutter is placed, which allows for rapid switching on/off the beam. Subsequently the polarizing cube beam splitters (PBSs), waveplates and mirrors are used to divide and steer the beam into three mutually perpendicular retro-reflected beams, of the same intensity and proper circular polarizations intersecting in the center of the chamber. For actual experiments with the cold atoms other ECDLs of our own construction equip- ped with adequate control systems are used, these are not included in the scheme of Fig. 11.

An important issue for trap functioning is the laser frequency stabilization and tuning. The lasers' linewidths are $<1 \mathrm{MHz}$, and the awaited frequency stability is of the same range at least in the data taking time. The respective system consists of two components. The first relies on the Doppler broadened method with the dichroic atomic vapor laser lock (DAVLL) scheme [40] applied, and the other on the Doppler free method with the frequency control unit based on the saturated absorption spectrometer (Fig. 12). Since DAVLL relies on the Doppler broadened transition for experiments with $\mathrm{MHz}$ resolution, the use of the second spectrometer is necessary to calibrate the laser frequency against ${ }^{85} \mathrm{Rb}$ hyperfine structure line positions. The trapping laser is actively locked to a respective crossover resonance of a transition in a saturated absorption spectrometer. Signal of both spectrometers are generated in their own $\mathrm{Rb}$ vapor filled glass cells and detected with photodiodes. To improve a control and precise setting of the trapping beam frequency the setup was equipped with an acousto optical modulator (AOM). A double pass AOM scheme was used between DL1 and DL2 lasers giving a sub-MHz precision and $40 \mathrm{MHz}$ tuning range (not shown in Fig. 11).

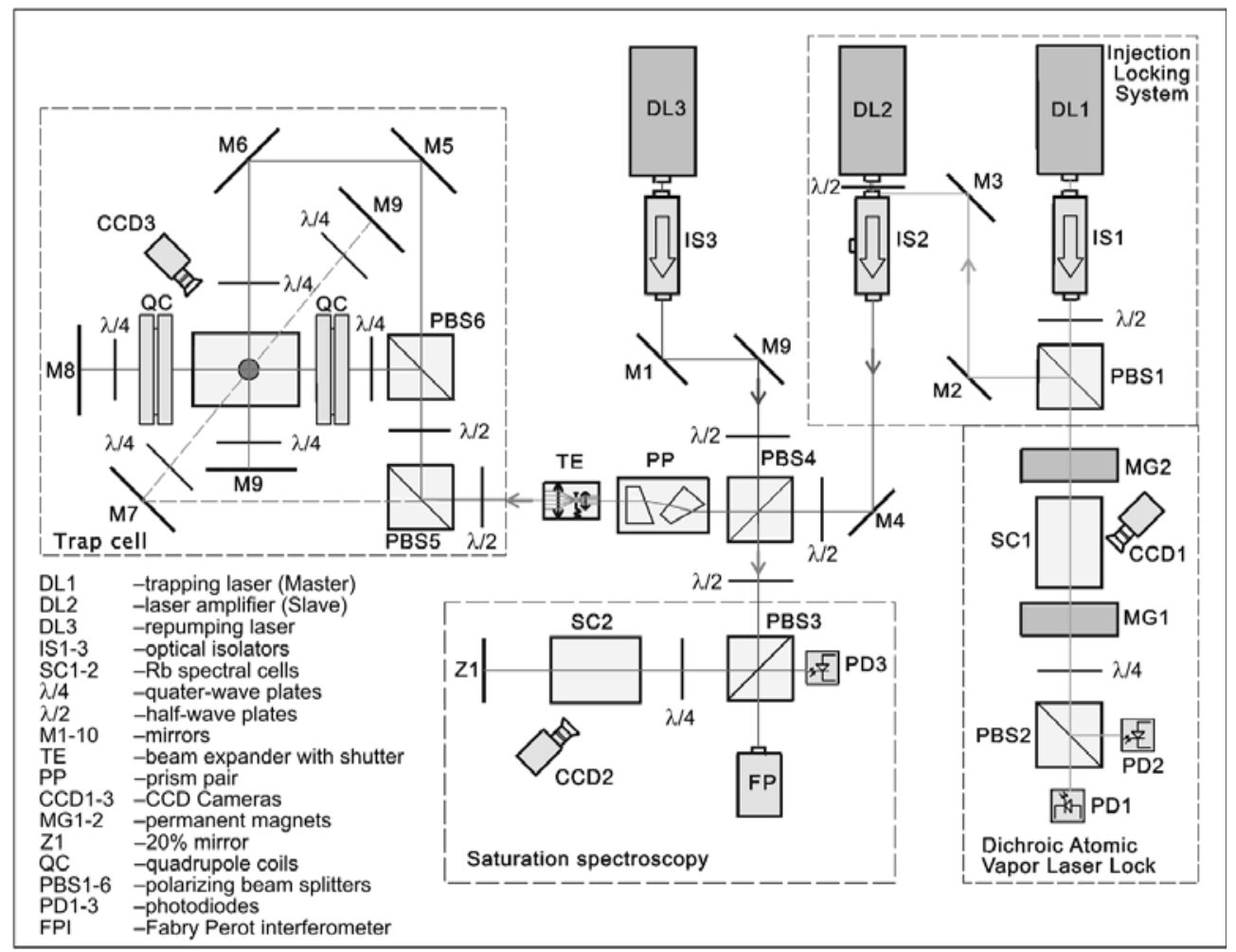

Fig. 11. Layout of the optical table with our MOT. Schematic beam traces of the trapping and repumping lasers are shown Essential blocks are marked with a dashed line 


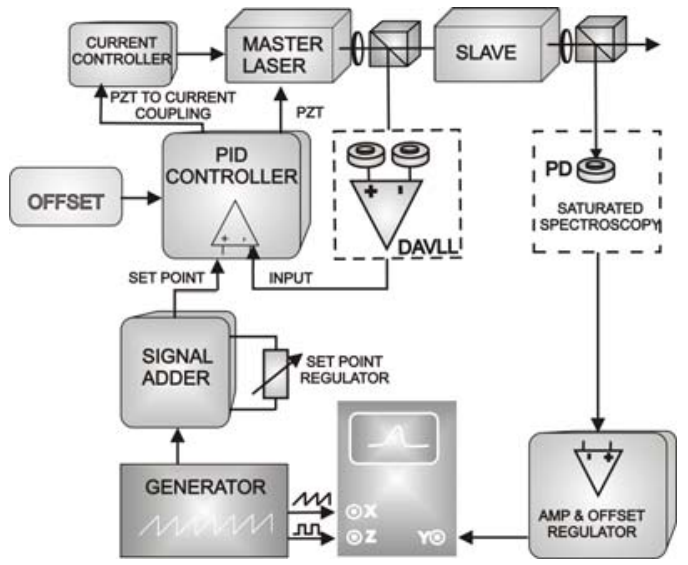

Fig. 12. Scheme of the system for trapping laser frequency stabilization and control

Two photographs of the optical table are shown. In Fig. 13(a) in the background at the central position the main part of the system with the MOT chamber can be seen. View of the chamber is screened by small coils (diameter of about $100 \mathrm{~mm}$ ) producing the inhomogeneous $B$ field. Some fragments of the big coils for cancellation of an earth and stray magnetic fields are also visible. In the foreground an extra (coupling) laser used in our experiments can be seen. In Fig. 13 (b) a general view of the laser system is shown. The MOT lasers are situated in the lower table together with the frequency stabilization arrangement. On the upper table an additional (probing) laser is placed.

In our MOT the steady state number of trapped atoms (given by the balance between loading and loss rates), amounts to $10^{6}-10^{8}$ depending on the trap parameters (laser intensity, detuning and magnetic field gradient) in a cloud of a size typically about $1 \mathrm{~mm}$ and the temperature of about
$100 \mu \mathrm{K}$. Some other details about our MOT can be found in an earlier publication [22].

\section{III.3. The apparatus for spectral studies}

The trapped atoms offer a convenient environment for a wealth of studies. Various processes have been studied with our apparatus by probing the cold sample with additional laser(s). The apparatus is aimed at registering laser induced spectra. During the data taking the MOT lasers can be constantly on or alternatively switched off. In the latter case one can avoid the perturbing influence of the MOT fields. However, with this approach the timing requirements are more stringent.

The control of many experimental parameters as well as the measurement taking demand a lot of care. To allow the smooth operation a computerized system was built. In Fig. 14 a diagram is shown of a system, dedicated for control of the experimental parameters and for data acquisition. The device (DMS2000) entirely constructed and programmed in our laboratory is based on the microcontroller (ATmega32) connected via USB port to a PC. It enables execution of a programmed sequence of experimental events including turning on/off the MOT fields, readouts of signals, advancing laser frequency after the end of the data acquisition period, etc. Due to application of the microcontroller, timing sequence can be established precisely, with $\mu$ s accuracy, which is usually not available in many measurement systems based on the Windows platform.

Below two examples of the high resolution pumpprobe measurements performed with our experimental arrangement in cold ${ }^{85} \mathrm{Rb}$ atoms are given.
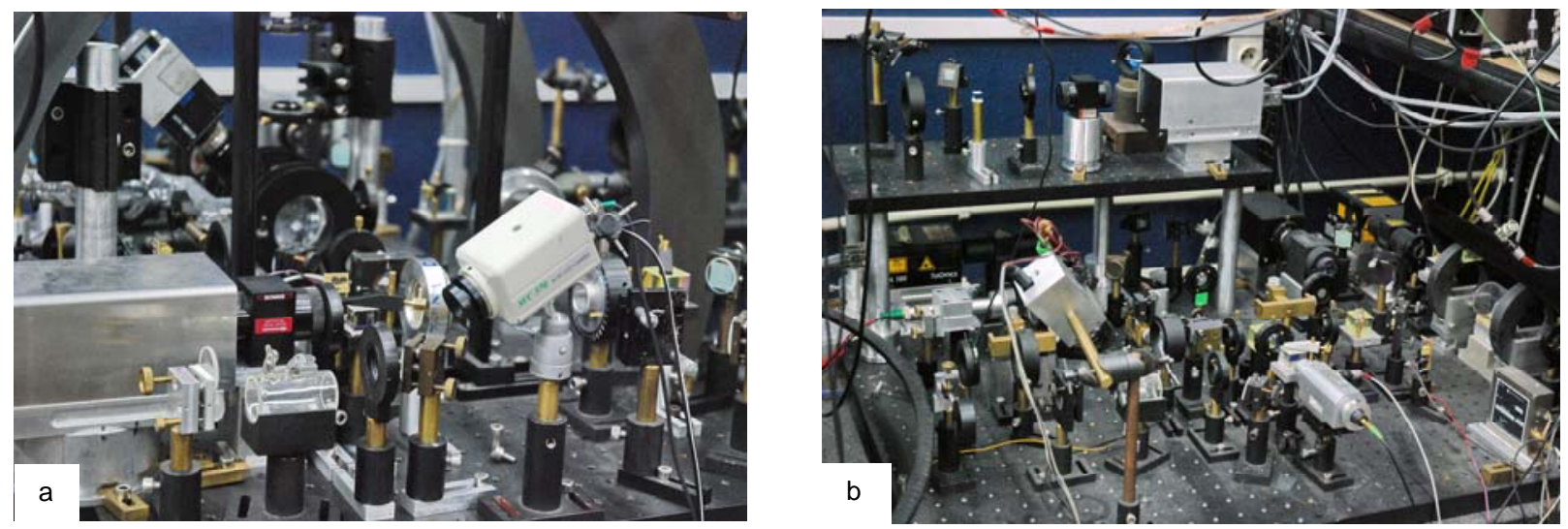

Fig. 13. Main areas of the setup: (a) In the foreground the coupling laser. In the background the area of the MOT chamber hidden within small coils. (b) The main part of the laser and frequency control arrangement. Lower table: the trapping and repumping lasers with accompanying systems. Upper table: the probing laser. The depicted system undergoes frequent modifications 


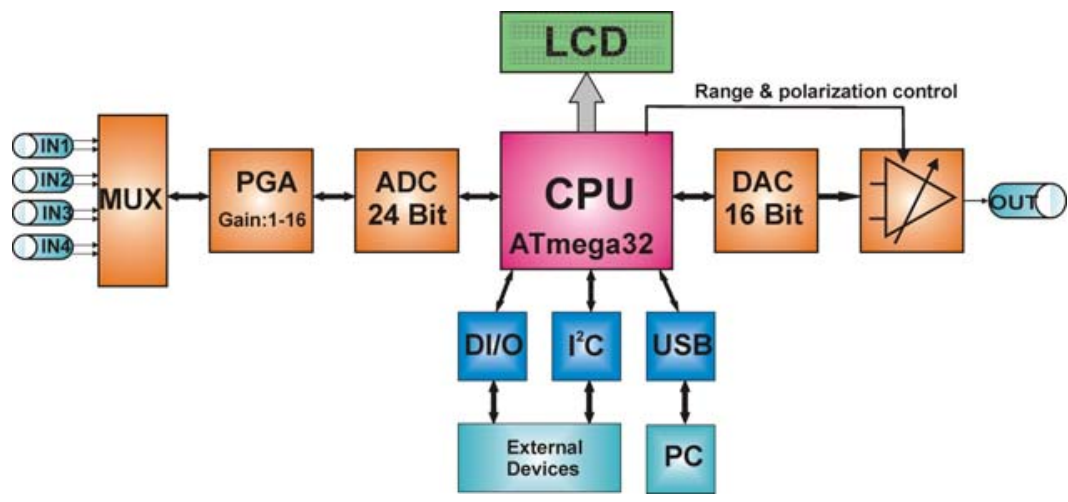

Fig. 14. The block diagram of the digital system for data acquisition, digital frequency tuning and the experiment control. MUX - 4 channel analog multiplexer; PGA - programmable gain amplifier; ADC - 24 bit analog to digital converter; CPU ATmega32 microcontroller; DAC - 16 bit digital to analog converter; DI/O - 4 digital input lines and 2 digital output lines; $\mathrm{I}^{2} \mathrm{C}$ - two wire bus; USB - universal serial bus; LCD - 4 line LCD display

\section{EXAMPLES OF THE SETUP PERFORMANCE}

\section{IV.1. Spectrum of the $5 \mathrm{~S}_{1 / 2}(F=3) \rightarrow 5 \mathrm{P}_{3 / 2}\left(F^{\prime}=2,3,4\right)$ transition}

Figure 15 shows the complex absorption spectrum of a weak probe $\left(I_{\text {probe }} \sim 1 \mu \mathrm{W}\right)$ tuned across the $5 \mathrm{~S}_{1 / 2}(F=3) \rightarrow$ $5 \mathrm{P}_{3 / 2}\left(F^{\prime}=2,3,4\right)$ transitions in a working MOT. As can be seen, the intense $\left(I_{\text {trap }} \sim 20 \mathrm{~mW} / \mathrm{cm}^{2}\right.$, detuning $\delta_{\text {trap }}=$ $-16 \mathrm{MHz}$ ) cooling field at the $5 \mathrm{~S}_{1 / 2}(F=3) \rightarrow 5 \mathrm{P}_{3 / 2}\left(F^{\prime}=4\right)$ resonance, modifies significantly the probed transition. The spectrum is characterized by a highly nonlinear character.

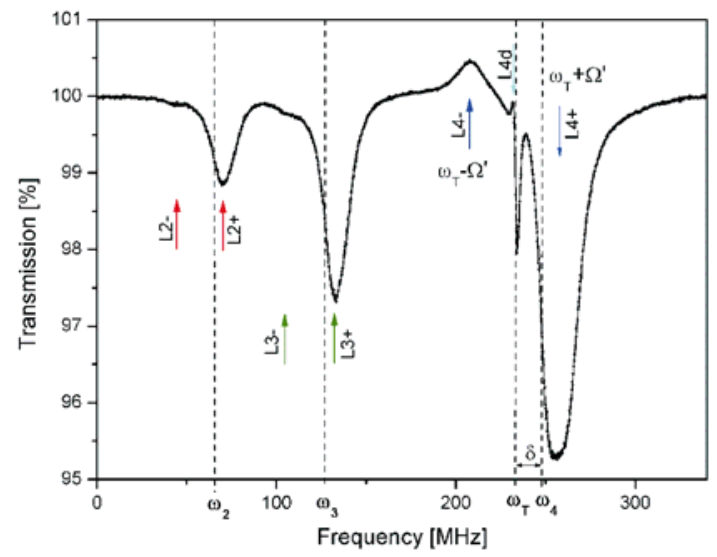

Rys. 15. Transmission of a weak probe beam across the ${ }^{85} \mathrm{Rb}$ atoms' cloud in a working MOT. Vertical dashed lines mark frequencies $\omega_{2}, \omega_{3}, \omega_{4}$ of the unperturbed $5 \mathrm{~S}_{1 / 2}(F=3) \rightarrow 5 \mathrm{P}_{3 / 2}\left(F^{\prime}\right.$ $=2,3,4)$ transitions. Arrows denoted with $L 2 \pm, L 3 \pm, L 4 \pm$ and $L 4 d$ point at the spectral features, resulting from trapping field induced modification of the levels. Trapping laser frequency $\omega_{T}$ and its detuning $\delta=-16 \mathrm{MHz}$ from the exact resonance $\left(\omega_{4}\right)$ are also shown. $\Omega$ ' is for generalized Rabi frequency
It reveals the Autler-Townes doublets (denoted as $L 2 \pm$ and $L 3 \pm$ ), the absorption $(L 4+)$ and gain $(L 4-)$ structures as well as the dispersionlike feature of subnatural linewidth $(L 4 \mathrm{~d})$. Note that that the Autler-Townes doublets' components are of significantly differing amplitudes which is a result of the off-resonant excitation of the strongly-driven (trapping) transition. Further explanation of the observed features is beyond the scope of this contribution. It can be found e.g., in $[41,42]$.

\section{IV.2 Study of the $5 P_{3 / 2} \rightarrow 5 D_{J}$ transition}

In Fig. 16 an example of an absorption spectrum in a multilevel cascade scheme with a dense hyperfine structure in the uppermost level is shown. In this experiment we sampled the ${ }^{85} \mathrm{Rb}$ MOT with a weak probe beam tuned across the $5 \mathrm{P}_{3 / 2}\left(F^{\prime}=4\right) \rightarrow 5 \mathrm{D}_{\mathrm{J}}\left(F^{\prime \prime}\right)(J=3 / 2,5 / 2)$ hyperfine manifolds, while the $5 \mathrm{~S}_{1 / 2}(F=3) \rightarrow 5 \mathrm{P}_{3 / 2}\left(F^{\prime}=4\right)$ transition was driven by the red-detuned trapping beam. The transmission spectra of this cascade system were registered for a number of detunings and intensities of the trapping field. The spectrum presented shows an example of absorption to the $5 \mathrm{D}_{3 / 2}\left(F^{\prime \prime}=3,4\right)$ manifold. The multiple peaks are due to both its hyper fine structure and the acStark (equivalentlyAutler-Townes) splitting in the first, lower step of the cascade $5 \mathrm{~S}_{1 / 2}(F=3) \rightarrow 5 \mathrm{P}_{3 / 2}\left(F^{\prime}=4\right) \rightarrow$ $5 \mathrm{D}_{\mathrm{J}}\left(F^{*}\right)$. Features denoted with $(*, * *)$ corespond to the Autler-Townes doubled hyperfine components. The spectra were simulated as a sum of Lorentzians via iterative fitting procedure. In this way the line positions and the separations between the doubled features were obtained. The performed measurements of the Autler-Townes splitting could be used for direct determination of the Rabi frequency $\Omega$ of 


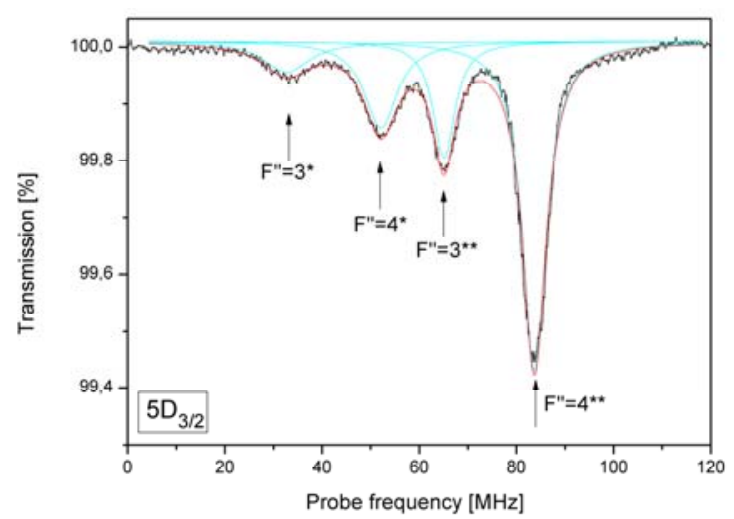

Fig. 16. Representative probe transmission spectrum in multilevel cascade scheme $5 \mathrm{~S}_{1 / 2}(F=3) \rightarrow 5 \mathrm{P}_{3 / 2}\left(F^{\prime}=4\right) \rightarrow 5 \mathrm{D}_{3 / 2}\left(F^{\prime \prime}=3,4\right)$. The probe is tuned across the $5 \mathrm{P}_{3 / 2}\left(F^{\prime}=4\right) \rightarrow 5 \mathrm{D}_{3 / 2}\left(F^{\prime \prime}=3,4\right)$ transition while the strong trapping laser excites the $5 \mathrm{~S}_{1 / 2}(F=3)$ $\rightarrow 5 \mathrm{P}_{3 / 2}\left(F^{\prime}=4\right)$ transition. The trapping field intensity $I_{\text {trap }}=22$ $\mathrm{mW} / \mathrm{cm}^{2}$ and detuning $\delta=-26 \mathrm{MHz}$. Features denoted with $(*, * *)$ see text

the strong trapping laser which is an important parameter of the trap. The results are depicted in Fig. 17, where the measured splittings are drawn for both hyperfine transitions $\left(F^{\prime}=4\right) \rightarrow\left(F^{\prime}=3\right)$ and $\left(F^{\prime}=4\right) \rightarrow\left(F^{\prime}=3\right)$ separately. Using the formula for the generalized Rabi frequency, for detuning $\delta$,

$$
\Omega^{\prime}=\sqrt{\Omega^{2}+\delta^{2}},
$$

and noting that the measured splitting is approximately equal to $\Omega$ ' we have calculated the value of Rabi frequency to be $\Omega=16.4 \pm 0.5$. More results and a detailed discussion are given in [43].

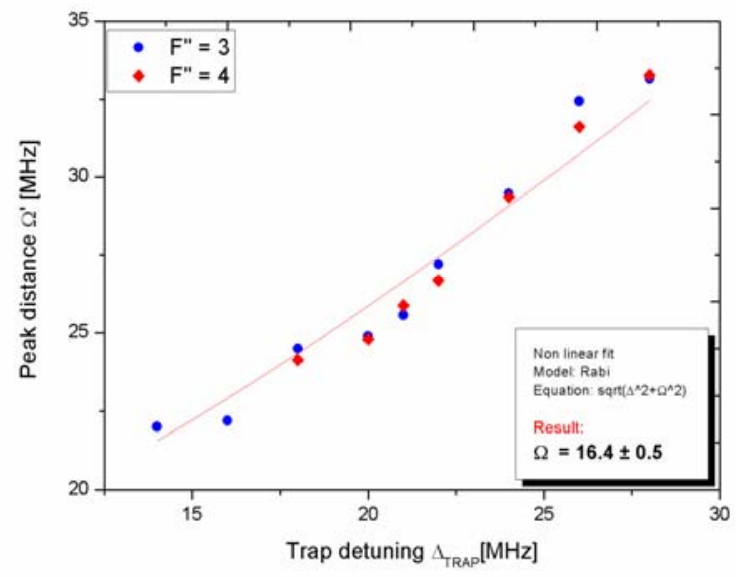

Fig. 17. The separation between the peaks of respective spectral features $v s$. trap detuning. Data were taken from a series of measurements of the type shown in Fig. 16. For all measurements in the plot the trapping intensity was kept constant $I_{\text {trap }}=22 \mathrm{~mW} / \mathrm{cm}^{2}$. The corresponding (approximate) Rabi frequency $\Omega=16.4 \pm 0.5$ was obtained as a parameter of the fit

\section{SUMMARY}

A brief overview of the laser cooling techniques was given with the aim to elucidate the working principles of the magneto-optical trap. The practical realization of the rubidium MOT at the IP PAS in Warsaw was briefly discussed. Some components were presented in more detail. Two of our experiments performed in a cold ${ }^{85} \mathrm{Rb}$ sample were chosen to illustrate the capabilities of the experimental arrangement.

\section{Acknowledgements}

M. Głódź, K. Kowalski, and J. Szonert gratefully acknowledge partial financial support of this work by the Polish Ministry of Science and Higher Education from the funds for science in the years 2007-2010. V. Cao Long, K. Dinh Xuan, and B. Nguyen Huy would like to thank for financial support from the Vietnam's Ministry of Science and Technology under the project coded 03/2009/HD-NDT.

\section{References}

[1] E.A. Cornell, C.E. Wieman, Nobel Lecture: Bose-Einstein Condensation in a Dilute Gas, the First 70 Years and Some Recent Experiments. Rev. Mod. Phys. 74, 875-893 (2002).

[2] V.I. Balykin, V.G. Minogin, V.S. Letokhov, Electromagnetic trapping of cold atoms. Rep. Prog. Phys. 63, 14291510 (2000).

[3] T.W. Hänsch, A. Schawlow, Cooling of gases by laser radiation. Opt. Comm. 13, 68-71 (1975).

[4] W. Phillips, H. Metcalf, Laser Deceleration of an Atomic Beam. Phys. Rev. Lett. 48, 596-599 (1982).

[5] S. Chu, L. Hollberg, J.E. Bjorkholm, A. Cable, A. Ashkin, Three-dimensional viscous confinement and cooling of atoms by resonance radiation pressure. Phys. Rev. Lett. 55 (1), 48-51 (1985).

[6] E.L. Raab, M. Prentiss, A. Cable, S. Chu, D. Pritchard, Trapping of Neutral Sodium Atoms with Radiation Pressure. Phys. Rev. Lett. 59, 2631 (1987).

[7] C.N. Cohen-Tannoudji, Manipulating atoms with photons, Nobel Prize Lecture. 8th December 1997.

[8] C. Monroe, W. Swann, H. Robinson, C. Wieman, Very Cold Trapped Atoms in a Vapor Cell. Phys. Rev. Lett. 65, 1571-1574 (1990).

[9] J. Zachorowski, T. Pałasz, W. Gawlik, Magneto-Optical Trap for Rubidium Atoms. Opt. Appl. 28, 239 (1998).

[10] S.G. Miranda, S.R. Muniz, G.D. Telles, L.G. Marcassa, K. Helmerson, V.S. Bagnato, Dark-spot atomic-beam slowing for on-axis loading of traps. Phys. Rev. A 59, 882 (1999).

[11] K. Dieckmann, R.J.C. Spreeuw, M. Weidemüller, J.T.M. Walraven, Two-dimensional magneto-optical trap as a source of slow atoms. Phys. Rev A 59, 3891 (1998).

[12] J. Schoser, A. Batar, R. Low, V. Schweikhard, A. Grabowski, Yu. B. Ovchinnikov, T. Pfau, Intense source of cold Rb atoms from a pure two-dimensional magneto-optical trap. Phys. Rev A 66, 023410 (2002). 
[13] M.S. Santos, P. Nussenzveig, L.G. Marcassa, K. Helmerson, J. Flemming, S.C. Zilio, V.S. Bagnato, Simultaneous trapping of two different atomic species in a vaporcell magneto-optical trap. Phys. Rev. A 52, 4340 (1995).

[14] J. Goldwin, S.B. Papp, B. DeMarco, D.S. Jin, Two-species magneto-optical trap with ${ }^{40} \mathrm{~K}$ and ${ }^{87} \mathrm{Rb}$. Phys. Rev. A 65, $021402(2002)$.

[15] M.O. Mewes, G. Ferrari, F. Schreck, A. Sinatra, C. Salomon, Simultaneous magneto-optical trapping of two lithium isotopes. Phys. Rev. A 61, 011403 (1999).

[16] H. Jelassi, B. Viaris de Lesegno, L. Pruvost, Photoassociation spectroscopy of ${ }^{87} \mathrm{Rb}_{2}\left(5 \mathrm{~S}_{1 / 2}+5 \mathrm{P}_{1 / 2}\right)$ Og long-range molecular states: Analysis by Lu-Fano graph and improved LeRoy-Bernstein formula. Phys. Rev. A 73, 032501 (2006).

[17] M.H. Anderson, J.R. Ensher, M.R. Matthews, C.E. Wieman, E.A. Cornell, Observation of Bose-Einstein Condensation in a Dilute Atomic Vapor. Science 269 (1995).

[18] C.J. Myatt, E.A. Burt, R.W. Ghrist, E.A. Cornell, C.E. Wieman, Production of Two Overlapping Bose-Einstein Condensates by Sympathetic Cooling. Phys. Rev. Lett. 78, 586 (1997).

[19] F. Bylicki, W. Gawlik, W. Jastrzębski, A. Noga, J. Szczepkowski, M. Witkowski, J. Zachorowski, M. Zawada, Studies of the Hydrodynamic Properties of Bose-Einstein Condensate of ${ }^{87}$ Rb Atoms in a Magnetic Trap. Acta Phys. Pol. A 113, 691 (2008).

[20] W.D. Phillips, Laser cooling and trapping of neutral atoms. Rev. of Mod. Phys. 70, 721-741 (1998).

[21] M. Gajda, J. Mostowski, 3-Dimensional Theory of the Magnetooptical Trap-Doppler Cooling in the Low-Intensity Limit. Phys Rev A, 49 (6), 4864-4875 (1994).

[22] K. Kowalski, E. Dimova-Arnaudova, K. Fronc, S. Gateva, M. Głódź, L. Lis, L. Petrov, J. Szonert, A system for magnetooptical cooling and trapping of Rb atoms. Opt. Appl. 36, 559-567 (2006).

[23] S. Stenholm, The semiclassical theory of laser cooling. Rev. Mod. Phys. 58, 699-739 (1986).

[24] H. Metcalf, P. van der Straten, Cooling and trapping of neutral atoms. Phys. Rep. 244, 204-286, (1994).

[25] H.J. Metcalf, P. van der Straten, Laser Cooling and Trapping of atoms. J. Opt. Soc. Am. B 20, 887-908 (2003).

[26] H.J. Metcalf, P. van der Straten, Laser Cooling and Trapping. Springer, New York (1999).

[27] C.S. Adams, E. Riis, Laser cooling and trapping of neutral atoms. Progr. in Quant. Electr, 21 (1), 1-79 (1997).

[28] A. Ashkin, Acceleration and Trapping of particles by Radiation Pressure. Phys. Rev. Lett. 24, 156 (1970).

[29] A. Ashkin, J.P. Gordon, Stability of radiation-pressure particle traps: an optical Earnshaw theorem. Opt. Lett. 8, 511-513 (1983).
[30] V. Zehnle, Doppler cooling to the recoil limit by means of sharp atomic transitions with controlled quenching. J. Opt. Soc. Am. B 20, 931 (2003).

[31] P.D. Lett, R.N. Watts, Ch. I. Westbrook, W.D. Phillips, P.L. Gould, H. Metcalf, Observation of Atoms Laser Cooled below the Doppler Limit. Phys. Rev. Lett. 61 (2) 169-172 (1988).

[32] J. Dalibard, C. Cohen-Tannoudji, Laser cooling below the Doppler limit by polarization gradients - simple theoretical models. J. Opt. Soc. Am. B 6, 2023-2045 (1989).

[33] P.J. Ungar, D.S. Weiss, E. Riis, S. Chu, Optical Molasses and Multilevel Atoms - Theory. J. Opt. Soc. Am. B 6, 20582071 (1989).

[34] R. Maruyama, R.H. Wynar, M.V. Romalis, A. Andalkar, M.D. Swallows, C.E. Pearson, E.N. Fortson, Investigation of sub-Doppler cooling in an ytterbium magneto-optical trap. Phys. Rev. A 68, 011403(1-4) (R) (2003).

[35] M. Kasevich, S. Chu, Laser Cooling Below a Photon Recoil With 3-Level Atoms. Phys. Rev. Let. 69, 1741-1744 (1992).

[36] A. Aspect, E. Arimondo, R. Kaiser, N. Vansteenkiste, C. Cohen-Tannoudji, Laser Cooling Below the One-Photon Recoil Energy by Velocity-Selective Coherent Population Trapping. Phys. Rev. Let. 61, 826 (1988).

[37] K.B. MacAdam, A. Steinbach, C.E. Wieman, A narrowband tunable diode laser system with grating feedback, and a saturated absorption spectrometer for $C s$ and Rb. Am. J. Phys. 60, 1098 (1992).

[38] C.J. Hawthorn, K.P. Weber, R.E. Scholten, Littrow configuration tunable external cavity diode laser with fixed direction output beam. Rev. Sci. Instrum. 72, 4477 (2001).

[39] X. Wang, X. Chen, J. Hou, D. Yang, Y. Wang, Side-mode injection locking characteristics of $150 \mathrm{~mW}$ AlGaAs semiconductor lasers. Opt. Comm. 178, 165 (2000).

[40] V.V. Yashchuk, D. Budker, J.R. Davis, Laser frequency stabilization using linear magneto-optics. Rev. of Sci. Instr. $71,341(2000)$

[41] M. Mitsunaga, T. Mukai, K.Watanabe, T. Mukai, Dressed atom spectroscopy of cold Cs atoms. J. Opt. Soc. Am. B13, 2696-2700 (1996).

[42] Y.-Ch. Chen, Y.-W. Chen, J.-J. Su, J.-Y. Huang, I.A. Yu, Pump-probe spectroscopy of cold ${ }^{87} \mathrm{Rb}$ atoms in various polarization configurations. Phys. Rev. A63, 0438089 (1-11) (2001).

[43] K. Kowalski, M. Głódź, J. Szonert, to be published.

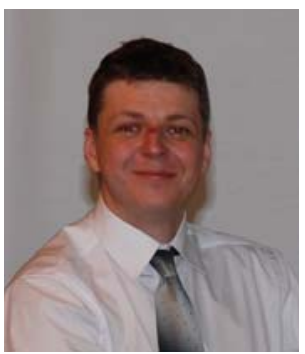

KRZYSZTOF KOWALSKI graduated from the College of Science in Warsaw with a MSc degree in experimental physics. His fields of interest cover cold atom physics, quantum and nonlinear optics and the design of digital measurement systems. He is the builder of Warsaw magneto-optical trap. In 2008 he defended his $\mathrm{PhD}$ thesis titled "Electromagnetically Induced Transparency in cold rubidium atoms in magneto-optical trap" at the Institute of Physics PAS, supervised by Assoc. Prof. M. Głódź. 


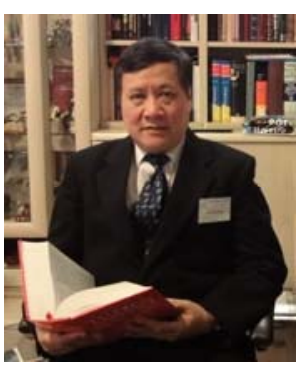

CAO LoNG VAN was born in 1952 in Hanoi, Vietnam. He graduated from Warsaw University in 1976 with an MSc degree in theoretical physics. His master thesis was entitled "Callan-Symanzik Equation and Transition Probabilities" and was written under the supervision of Prof. Iwo Białynicki-Birula. In 1979, he defended his $\mathrm{PhD}$ dissertation entitled "Phase Representation in Quantum Optics", also supervised by Prof. I. BiałynickiBirula. He habilitated in 1987 in the Center of Theoretical Physics, Polish Academy of Sciences in Warsaw with a thesis entitled "Noises in Quantum Optics". His research interests concern several topics in Quantum and Nonlinear Optics.

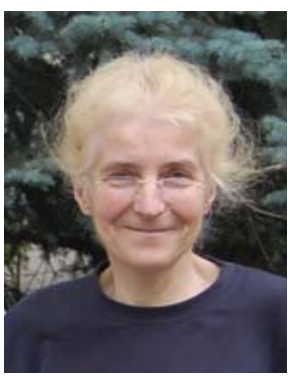

Malgorzata GıóDź received the MSc degree from the Warsaw University and she gained her PhD and habilitation at the Institute of Physics of the Polish Academy of Sciences (IP PAS) in Warsaw. She is working at IP PAS at a position equivalent to Assoc. Prof. For two years she was also doing research in Canada, as a guest of the Department of Physics of the University of Windsor. Her main scientific interests concentrate on atom-laser-field interactions. She dealt with experiments on the statistical properties of laser light and HiRes (quantum-beat) $h f_{s}$ spectroscopy, in collaboration with the Department of Physics of Warsaw University. In Canada, and then also in Poland, she studied atomic deexcitation processes, spontaneous and in thermal collisions. Currently, she is investigating cold atoms (coherent processes, HiRes spectroscopy) in a magnetooptical trap.

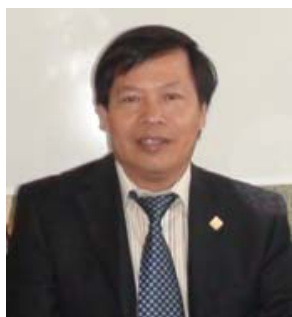

Dinh Xuan KhoA was born in Nghe An province, Vietnam. He graduated, with a major in Physics, from Vinh University in 1981. He completed a PhD course in Quantum Optics in 1996. His doctoral thesis entitled "Physics of Dye Lasers" was supervised by Prof. Cao Long Van and Prof. Dao Xuan Hoi. Since 1997, he has been the head of research group Optics Group in Vinh University. He was elected as Associate Professor in Physics in 2003. His field of interests covers a large variety of topics in Quantum and Nonlinear Optics, Atomic and Molecular Physics.

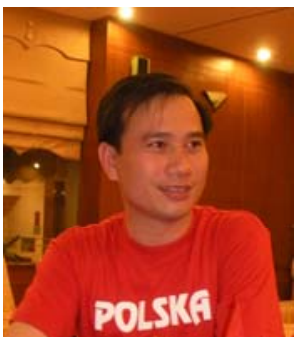

NGUYEN HUY BANG was born in Ha Tinh province, Vietnam. He graduated, in physics, from Vinh University in 2002. In 2004 he did PhD study at the Institute of Physics, Polish Academy of Sciences, under the supervision of Prof. W. Jastrzębski. Here he did research on alkali-metal diatomic molecules by using polarization labelling spectroscopy technique. After his graduation in Poland in 2008, he returned to work as lecturer in Optics Group, Faculty of Physics, Vinh University. Presently, his field of interest concerns physics of atoms, molecules, and optics.

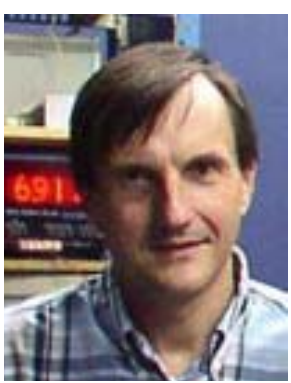

JERZY SzONERT graduated from Warsaw University with a MSc degree in experimental physics. His field of interest covers various topics of Spectroscopy, Nonlinear and Quantum Optics. Currently he is involved in experiments on cold atoms in the group of Assoc. Prof. M. Głódź. He is working in the Institute of Physics, Polish Academy of Sciences in Warsaw. 